\section{$\underset{\substack{\text { hommes } \\ \text { \& migrations }}}{ }$}

\section{Hommes \& migrations}

Revue française de référence sur les dynamiques

migratoires

$1301 \mid 2013$

Migrations et mondes ruraux

\title{
Futur simple et futur compliqué
}

Les Travailleurs étrangers en transit dans la roue saisonnière espagnole

\section{Emmanuelle Hellio}

\section{(2) OpenEdition}

12 Journals

\section{Édition électronique}

URL : http://journals.openedition.org/hommesmigrations/1896

DOI : 10.4000/hommesmigrations.1896

ISSN : 2262-3353

Éditeur

Musée national de l'histoire de l'immigration

\section{Édition imprimée}

Date de publication : 1 janvier 2013

Pagination : 19-27

ISBN : 978-2-919040-21-6

ISSN : 1142-852X

\section{Référence électronique}

Emmanuelle Hellio, «Futur simple et futur compliqué », Hommes \& migrations [En ligne], 1301 | 2013, mis en ligne le 31 décembre 2015, consulté le 20 avril 2019. URL : http://journals.openedition.org/ hommesmigrations/1896; DOI : 10.4000/hommesmigrations.1896 


\section{FUTUR SIMPLE \\ ET FUTUR COMPLIQUÉ \\ LES TRAVAILLEURS ÉTRANGERS EN TRANSIT DANS LA ROUE SAISONNIĖRE ESPAGNOLE}

Par EMMANUELLE HELLIO, doctorante en sociologie,

laboratoire Urmis, université de Nice

Pour les travailleurs migrants saisonniers, les saisons sont des frontières. Elles déterminent où et quand aura lieu le travail temporaire qui seul légitime leur présence. Dans le sud de l'Espagne, la cueillette des fraises constitue une étape dans la roue saisonnière qui commande les déplacements des migrants originaires d'Afrique noire, du Maghreb ou d'Europe de l'Est. Mis en concurrence, les travailleurs saisonniers, qu'ils soient titulaires d'un contrat de travail ou sans-papiers, peinent à imaginer sereinement l'avenir.

\section{Des Maliens assis sur des bidons autour du feu se penchent sur le livre de grammaire espagnole que la Croix-Rouge leur a distribué : Conjugue le futur simple. Ils rient. "Et même le futur compliqué. Ici c'est futur compliqué !"1}

La ruralité a longtemps constitué un objet d'étude marginal pour la sociologie française des migrations. Pourtant, les zones agricoles à forte population étrangère ne correspondent pas à l'idée archaïque et traditionaliste qu'on pourrait en avoir. Au contraire, intégrées dans des chaînes agro-alimentaires mondialisées, tant du point de vue des firmes multinationales qui les contrôlent, des techniques de culture, des pays de production et d'exportation que des travailleurs qui récoltent, ces zones sont de bons prismes pour observer les mécanismes contemporains de la globalisation agricole et migratoire.
La fraisiculture andalouse constitue une illustration fidèle du modèle californien décrit par JeanPierre Berlan en 1986. Dans cette zone de monoculture intensive située à Huelva, l'importation sous contrats temporaires de femmes des pays de l'Est et du Maroc à partir de 2000 a fait l'objet de nombreuses recherches ${ }^{2}$. Cette féminisation du recrutement est liée à la mise en œuvre d'un programme de migration temporaire, la contratación en origen $^{3}$. L'arrivée de femmes polonaises, roumaines puis marocaines a donné un tout nouveau visage à la main-d'œuvre présente dans les serres, auparavant surtout composée d'hommes souvent sans 
papiers, venus d'Afrique noire ou du Maghreb. C'est ce dont témoigne cet agriculteur interrogé au milieu des années 2000 par Alicia Reigada : “J'ai eu de tout, des Marocains, des Morenitos ${ }^{4}$ du Niger, de Côte d'Ivoire, du Sénégal, des

Le développement Équatoriens, des Argentins, des des contrats pour les Colombiens, de tout, mais cétait femmes s'est accompagné

d'un contrôle plus

important de l'embauche

de sans-papiers par les autorités pour combattre cette mobilité antérieure auto-organisée. des gens qui venaient et toi tu étais obligé de les faire travailler illégalement parce que, voilà, la fraise tu dois la ramasser 5 ." La contratación en origen n'est donc pas à l'origine des migrations dans la zone. Elle constitue une réponse des institutions et des organisations patronales à la venue de flux qu'elles estimaient ne pas contrôler suffisamment en permettant, dans ce secteur où le besoin de main-d'œuvre est temporaire, de "programmer [la migration] de manière à ce que le travailleur saisonnier ne reste dans notre province que le temps que dure la campagne et qu'il a du travail ${ }^{6 "}$.

La contratación en origen permet de privilégier le recrutement de travailleuses ayant des charges familiales au pays pour s'assurer de leur retour en fin de saison et donc concilier les besoins du marché du travail agricole et la volonté étatique de limiter l'installation des étrangers. Le développement des contrats pour les femmes s'est accompagné d'un contrôle plus important de l'embauche de sans-papiers par les autorités pour combattre cette mobilité antérieure auto-organisée. "Des petits flux qui rentraient sans qu'on sache comment, on est passé à l'idée de ne plus importer tout le personnel qui le désirerait mais de sélectionner à la source ${ }^{7}$, résume le chef de culture d'une grande ferme. La mise en œuvre des contrats et la légalisation de la force de travail qui en a découlé ont davantage permis de choisir le

profil et de contrôler le temps de présence des travailleurs que de défendre leurs droits.

Ce remplacement de travailleurs sans papiers par la contratación en origen n'a eu lieu massivement quà Huelva car il correspond aux impératifs productifs de cette récolte qui dure quatre à cinq mois pendant lesquels l'agriculteur doit pouvoir compter sur une main-d'œuvre "en stock" ${ }^{8 ”}$, c'est-à-dire disponible sans qu'elle se voie pour autant assurer une activité continue. En Espagne, d'autres zones de production comme Almería continuent d'employer principalement une main-d'œuvre en illégalité. "Là-bas, il y a du travail et surtout ils ne considèrent pas les papiers ${ }^{9}$."

\section{Huelva au sein de la roue saisonnière}

Avant cette reconfiguration du recrutement, la saison de Huelva constituait un sas permettant aux sans-papiers d'Espagne de survivre et de travailler pendant les trois années de présence exigées par la ley de extranjería pour pouvoir prétendre à une carte de séjour. On y cherchait un employeur acceptant de signer un contrat d'un an afin d'être régularisé via la procédure d'arraigo social ${ }^{10}$. Le développement de la contratación en origen et l'aggravation de la crise économique ont rétréci ce sas en compliquant l'accès au contrat, et le chemin qui conduit à la régularisation s'est allongé indéfiniment.

À travers les expériences de deux hommes rencontrés en 2009 et retrouvés à Huelva trois années de suite, nous essaierons de voir quelle place la saison des fraises occupe dans leur parcours migratoire. Mohamed, Marocain, est venu du Royaume-Uni en Espagne en 2008 pour y "trouver les papiers ${ }^{11}$ ". Daouda, Burkinabé entré avec un visa touristique

\footnotetext{
4. Les employeurs nomment généralement les Africains "Negritos" ou "Morenitos". Le diminutif qui indique la compassion et l'affection s'inscrit dans les codes du racisme colonial qui différencie les Morenos, les Noirs, naïfs, dociles et infantiles, des Moros, les Arabes, considérés comme conflictuels. Voir Olivier Le Cour Grandmaison, Coloniser, exterminer. Sur la guerre et l'État colonial, Paris, Fayard, 2005. 5. Alicia Olaizola Reigada, art. cit., p. 170, traduit par l'auteur. 6. Projet AENEAS CARTAYA, “Gestion éthique de l'immigration de travail stable et temporaire", 2006, p. 18, traduit par l'auteur. http://www.ayto-cartaya.es/. 7. Entretien, 22 juillet 2010 , Moulay Bouslem, traduit par l'auteur. 8. Expression empruntée à Patrick Herman, Les Nouveaux Esclaves du capitalisme, Paris, Au diable Vauvert, 2008, p. 219). 9. Entretien avec Issa, 5 mai 2010, Chabola de la madre, Palos de la Frontera. 10. Un étranger prouvant qu'il se trouve en Espagne depuis trois ans et qu'un employeur lui propose un contrat d'un an obtient une carte de séjour et de travail d'un an. Article 31.3 de la loi sur les étrangers 4/2000. 11. Toutes les citations non référencées sont issues du carnet de terrain.
} 
en Allemagne en 2009, est descendu en Andalousie dès son arrivée car tout le monde lui disait: "En Espagne, il y a beaucoup d'argent, tu arrives là-bas, le lendemain tu travailles." À travers leurs récits se dessinent la rareté du travail, le racisme de la société rurale "d’accueil", les trafics qui se développent autour des contrats et l'espoir de la régularisation à venir.

Les parcours de ces deux hommes sont abordés à travers le prisme de la saison des fraises, mais ce n'est pour eux qu'une étape dans une roue saisonnière qui continue avec la cueillette des fruits à Lérida, des oranges à Séville ou des olives à Jaén.

"Moi pour l'instant je ne suis pas fixe, affirme Daouda, je suis là où il y a les campagnes ${ }^{12}$. [...] À Albacete, la campagne dure quatre mois de juin à septembre. Ensuite je suis allé à Jaén. [...] Là-bas la campagne peut durer trois mois si tu as de la chance et que tu tombes sur quelqu'un qui a un grand campo ${ }^{13}$. Sinon c'est deux mois, un mois, quinze jours. Ils fatiguent les gens et ensuite ils prennent des nouveaux qui ne sont pas fatigués. Vu l'argent que tu as dépensé pour venir et vivre là-bas, tu ne sors pas de bénéfices. À Jaén j’ai travaillé quinze jours. Et puis ensuite, j'ai fait la fraise : je suis arrivé en janvier 2009, à cette époque-là il y a juste la fraise. Après la fraise, c'est Lérida, la pomme, c'est ce quion cherche maintenant. Mais si les fraises n'ont pas bien donné, ça va être pareil là-haut. Il y a Albacete aussi qui doit commencer, mais Lérida c'est mieux, j’ai déjà fait Albacete mais pas Lérida, je veux savoir quelle souffrance il y a là-bas ${ }^{14}$." À chaque étape de leur itinérance, ces hommes cherchent à travailler afin d'être régularisés. Après un long parcours de clandestinité, ils sont arrivés à destination, mais ils rencontrent de grandes difficultés à se faire embaucher. Du fait de l'utilitarisme migratoire qui conditionne le séjour au travail et de la crise économique qui empêche d'en trouver, Huelva et les autres campagnes espagnoles se sont transformées en frontières juridiques presque impossibles à traverser pour des hommes et des femmes maintenus en dehors d'une existence légale et qui s'épuisent petit à petit de ne pas y arriver: "Ce n'est pas l'Europe ça, l'Europe ça commence plus haut", se plaint Daouda. D'autres montrent les

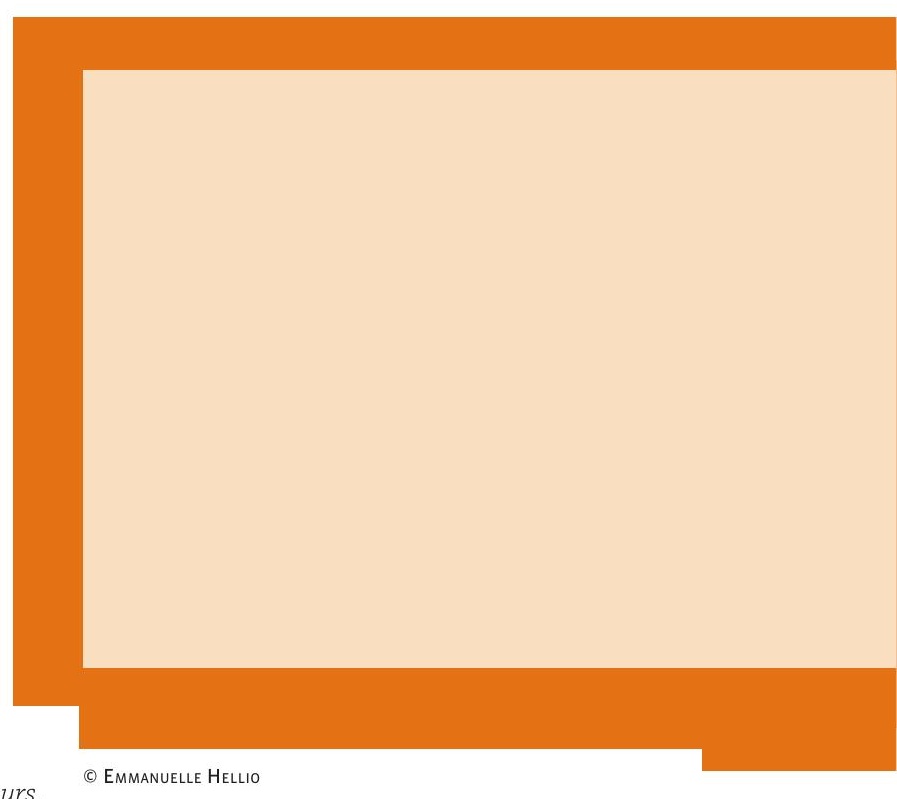

cabanes où ils sont regroupés : "C'est l'Europe ça ? On dirait la brousse." Ces campements dans lesquels ils se regroupent aux abords des serres sont la continuité des mises à l'écart que connaissent les étrangers regroupés dans des campements construits aux portes de l'Europe. Ici, comme là-bas, leur précarisation profite à un système productif.

Dans un tel contexte, lorsqu'ils ont l'occasion de travailler, ces ouvriers cherchent à se détacher du lot pour obtenir un contrat d'un an en travaillant le plus vite et le mieux possible, alors même que la mise en compétition des travailleurs selon le sexe, la nationalité et le statut juridique impose un rendement très élevé. Le pouvoir des employeurs sur la frontière donne ainsi forme aux relations de travail. 
Principales campagnes agricoles évoquées par mes interlocuteurs, tiré du calendrier de travail de l'Union générale des travailleurs. http://europadirectoandujar.blogspot.fr/p/empleoagricola-2012.html, consulté le 3 septembre 2012.

\section{Le pouvoir de l'employeur sur la frontière}

Depuis la généralisation de la contratación en origen, les employeurs préfèrent recruter les femmes étrangères sous contrat. Elles sont en situation légale, sont considérées comme plus adaptées à la récolte qui demanderait des compétences supposées féminines (capacité à se courber, patience, habileté) et on peut compter sur elles jusqu'à la fin de la saison puisqu'elles n'ont pas d'autres alternatives d'emploi. "Ils partent toujours trop tôt", se plaint Manolo, qui exploite dix hectares de serres, alors que, pendant l'entretien, nous sommes interrompus trois fois par des Africains venant chercher le solde de leur salaire avant de partir pour Lérida. "Je vais te dire une chose, ils vont se retrouver sans travail et là il y aura un problème. Moi je les considère tous très bons. [...] Mais ils partent avant la fin de la saison, ils te laissent tomber et partent à Léri$d a^{15}$." Devant ces propos, Daouda reconnaît: "C'est ce qui se dit ici : 'Un Noir, ça ne sert à rien, [...] ça prend les papiers et ça s'en va.' [...] C'est notre défaut aussi tu vois. Mais d'un autre côté, il ne faut pas nous accuser parce qu'on est venus pour chercher de l'argent."

Dans l'esprit des employeurs comme des saisonniers, ceux qui ont obtenu leurs papiers grâce au contrat fait par un agriculteur lui sont redevables, et lorsqu'ils dérogent à leurs obligations, ce n'est 
pas sans culpabilité. Kacim a été régularisé par un patron de Huelva en 2010. "Cette année, quand je suis revenu, je suis allé travailler pour un autre patron qui offrait plus d'heures de travail. J'ai appelé le chef qui m'a fait les papiers pour lui dire que cette année, je ne pouvais pas travailler pour lui parce que je rentrais en Afrique. Je l'ai croisé l'autre jour dans les rues de Moguer. Il ne m'a rien dit et m'a même invité à la romería (fête du village). J'avais un peu honte et ensuite j'ai menti encore une fois. Je lui ai dit que j'avais changé de chef parce que celui-là me payait 40 euros la journée et qu'il y avait beaucoup d'heures supplémentaires. Ce n'est pas vrai, en fait, c'est juste que je ne veux pas travailler pour lui parce qu'il cultive la framboise et il y a peu de travail ${ }^{16}$."

Dans une certaine mesure, en assurant à long terme une captivité de la main-d'œuvre, la contratación en origen répond aux inconvénients du système de mobilisation antérieur qui n'était pas suffisamment durable : après les trois ans d'illégalité, la régularisation des travailleurs les libérait. Ainsi, depuis la généralisation du recrutement sous contrat temporaire, les agriculteurs ne font appel aux Subsahariens et aux Maghrébins que pour les tâches qu'ils estiment masculines, lorsqu'il y a des pics d'activité ou qu'il faut remplacer des travailleurs. "Je ramasse dans un endroit où je ne faisais que la plantation, parce que le patron disait toujours la même chose: 'Pour la récolte, un Moreno ça ne va pas. Pour la plantation si, parce que les Noirs sont forts, mais pour ramasser, le Moreno ne vaut rien.' Et maintenant, toutes les Roumaines qui travaillaient pour lui sont parties parce que le travail est trop dur, alors il nous a pris : on est cinq Blacks et il y a deux Marocaines. [...] À la fin de la saison, tu peux démonter les serres. Ça ils ne contrôlent pas parce que ça demande beaucoup de gens et puis avec du vent bon, il faut beaucoup de personnes. Oui, si tu as vraiment de la chance, tu peux avoir ça, pour dix jours." Ces hommes représentent aussi une alternative pour les employeurs qui, n'étant pas en règle avec la Sécurité sociale ou le Trésor public, n'ont pas accès à la contratación en origen. Manolo embauche ainsi plus de 80 \% d'Africains. "Au mois de sep- tembre, les saisons se terminent au Nord, et ils commencent à venir. Je sais qu'ils vont revenir, ce sont toujours les mêmes, alors je compte un peu sur eux ${ }^{17}$." Pour les agriculteurs les plus proches, les campements représentent des viviers où ils peuvent recruter des travailleurs sans toujours leur fournir de logement: "Le patron qui m'a fait travailler [...] il m'a pas logé, je suis toujours dans mon coin. Il dit que pour moi c'est temporaire." Pour autant, nombreux sont ceux qui ne parviennent pas à obtenir une seule journée de travail. En 2010, Nourelaï m'avait dit : "Huelva ça ne vaut rien, tous les jours tu cherches du travail et il n'y a pas de travail [...]. En 2008, je n'ai pas gagné le travail, 2009 pas de travail, 2010 pas de travail. J'arrive en mars et je repars en juin."

\section{Les logiques de discriminations}

Le recrutement de ces étrangers est justifié par des discours racistes diffusés aussi bien par les employeurs que par les travailleurs. Si on considère qu'ils ne sont pas assez canalisables, les Noirs sont cependant réputés pour être de bons travailleurs. Cette anecdote rapportée par le recruteur d'une coopérative patronale donne un bon aperçu des représentations qui régissent l'organisation du travail dans les champs: "Un jour, un patron m'appelle et me dit qu'il a un problème avec des Noirs dans sa ferme. Je lui dis: 'Écoute, ces Noirs ce n'est pas moi qui te les ai amenés. Qu'est-ce que j’ai à voir là-dedans ?' Il me répond: 'Non mais tu m'as amené des Roumaines et le problème c'est avec elles.' Alors je vais là-bas, et il me dit que les Noirs ne ramassent pas bien. Normalement les Noirs ramassent plus vite que les Roumaines. On va dans le champ, et ils allaient tous en ligne, et les Noirs avançaient au même niveau que les Roumaines. Et je vois qu'elles sont en minishort, avec un décolleté jusquelà sans soutien-gorge. [...] Je me tourne vers le chef et je lui dis : 'Comment veux-tu que tes Noirs avancent s'ils sont à côté de femmes habillées comme ça ?' On a parlé avec elles, et elles ont boutonné leur chemise et ils 
ont continué à travailler ${ }^{18}$." Commentant cette anecdote, Daouda confirme: "Lui il a pris les Morenos parce qu'ils courent plus vite, mais si tu les mets dans la même serre, il faut te dire qu'ils ne vont pas partir. Si tu mets Moreno/Roumaine/Moreno, elles vont critiquer les Noirs et dire: 'Quand c'est fini, tu vas où ${ }^{19}$ ?' Ils ne vont pas avancer. Si tu mets

Les Roumainssont les Roumaines ensemble beaucoup moins dépendants du bon-vouloir des patrons que les travailleurs sous contrat ou sans papiers depuis que la Roumanie a commencé à intégrer

I'Union européenne et qu'ils jouissent d'une plus grande liberté de circulation. et les Morenos à côté, moi je vais vouloir faire la course. Mais si tu nous mélanges on va causer. En plus, c'est des femmes, c'est une causerie intéressante. Quand on ramasse, les Roumains nous disent de ralentir, mais nous on peut pas parce que les patrons savent qu'on ramasse plus vite, alors si tu baisses, tu vas avoir des problèmes." Les employeurs font aussi appel à eux pour menacer les autres travailleurs d'un possible remplacement. En juin 2011, alors que des Marocaines s'étaient mises en grève en refusant d'être illégalement payées au rendement, l'employeur les a immédiatement menacées de les remplacer par des Africains. Habiba se rappelle: "On a toutes décidé de ne pas travailler pour 1 euro la caisse. Il a dit : 'D'accord, moi je vais aller chercher des Noirs qui vont travailler.' Il prenait son téléphone pour nous montrer qu'il était prêt à appeler ${ }^{20}$."

Pour comprendre cette mise en compétition, justifiée ici par des particularités qui seraient propres à chaque groupe ethnique ou à chaque sexe, il faut prendre en compte les différences de statut juridique des groupes de travailleurs. Les Roumains sont beaucoup moins dépendants du bon-vouloir des patrons que les travailleurs sous contrat ou sans papiers depuis que la Roumanie a commencé à intégrer l'Union européenne et qu'ils jouissent d'une plus grande liberté de circulation. Face aux difficultés rencontrées par les travailleurs des pays tiers pour trouver un contrat d'un an, certains pré- fèrent l'acheter. Un contrat coûte entre 1000 et 1500 euros selon la période de l'année. Les prix baissent lorsque les agriculteurs ont besoin de liquidités et augmentent lorsqu'ils obtiennent les prêts leur permettant de financer la plantation. On peut également acheter pour 200 à 300 euros des certificats médicaux antidatés qui permettent à celui qui veut déposer un dossier d'arraigo social de le faire avant les trois ans réglementaires. Enfin, pour trouver plus facilement du travail, il y a la possibilité de se faire prêter ou de louer des papiers. Malgré tout, depuis 2010, les autorités contrôlent davantage la régularisation par arraigo social. Il est désormais exigé que l'employeur accompagne son travailleur pour accorder l'autorisation de travail et on vérifie systématiquement si l'année précédente, il avait déclaré un travailleur à l’année.

\section{La chabola comme refuge}

Dans la pratique, ce flux de travailleurs qui ne parvient pas à se faire embaucher se regroupe dans des campements construits au milieu des serres. Ces dernières années, les chabolas se sont enfoncées dans les forêts. On ne retrouve plus des dizaines d'Africains dormant le soir de leur arrivée dans les rues des villages sur leurs grosses valises à roulettes. Le logement collectif dans les fermes ou dans les chabolas crée une séparation entre la population autochtone et les étrangers. Même s'ils se rendent dans les villages à pied ou en taxi clandestin pour y faire des courses, ils se tiennent à l'écart de certains lieux. "Ici il y a des bars où un chien peut entrer, mais le Moro n'entre pas", me dit Mohamed. Daouda, lui, a tendance à éviter les interactions avec la population locale: "Si tu vas avec les Blancs, ils veulent toujours te montrer ta peau. [...] Si tu n'es pas courageux, tu ne vas pas avec eux. Moimême je ne trouve pas tellement le courage, jévite un peu. Grâce à Dieu il n'y a pas atteinte physique mais dans les yeux, tu vois que le gars peut te tuer." Leur 
darité et des liens faibles permettent à ces communautés transitoires de survivre dans des contextes hostiles ${ }^{22}$. Ces travailleurs itinérants développent un savoir-faire qui rappelle la culture de la mobilité créée par les hobos aux États-Unis à la fin du XIX siècle $^{23}$. Selon ses habitants, la chabola de la madre existe depuis 2004. Ce campement ressemble à un village. En 2010, j'y ai compté 49 abris

(c) Emmanuelle Hellio

présence est tolérée en proportion de leur discrétion. Lorsqu'ils se mobilisent pour réclamer du travail, les représailles sont immédiates. En 2009, des manifestations organisées par le Sindicato Obrero del Campo ont entraîné des destructions de campements à la suite de réunions entre les organisations agraires, les maires, les agriculteurs, la police et les ONG. Lors d'une de ces destructions, un brigadier, à qui je faisais remarquer que les habitants de la chabola détruite étaient déjà en train de reconstruire d'autres cabanes derrière la colline, m'a rétorqué : "Ça ne me dérange pas ça. Ils sont peutêtre même mieux là-bas. L'important, c'est qu'ils ne soient pas trop ensemble, qu'ils soient dispersés. Ça ne me dérange pas qu'il en reste quelques-uns ${ }^{21}$." Ainsi, la présence, même durable, est acceptée tant qu'elle garde l'apparence du provisoire. Mohamed, qui a vécu trois ans dans la même cabane, affirme qu'il n'a jamais eu de problèmes avec la police: "Si un mec a un $4 x 4$ [...] ils vont dire : Regarde le Moreno. [...] Mais là ils ne nous voient pas. On est à notre place. [...] On ne peut pas construire de meilleures maisons, parce que sinon ils vont les détruire. Là avec une maison comme ça, ils savent que tu ne restes pas longtemps. Ils savent qu'un jour tu vas partir. Comme un chien pour aller fouiller d'autres poubelles." Malgré tout, les chabolas, et cette vie qui s'élabore dans l'épaisseur de la frontière intra-européenne, sont le terreau de nouvelles formes sociales. Une solide cuisines, 195 cabanes, 33 abris pour prendre des douches et 12 cabanes visiblement abandonnées. En 2009, il y avait également une mosquée en plastique et ses habitants s'étaient cotisés pour avoir l'eau courante. "Chacun a donné 50 centimes, 1 euro. Mais on était beaucoup, tout le monde est déjà parti. On était un village. Ici, même s'il n'y a pas de travail, c'est mieux qu'à Lérida ou Jaén où on dort dans la rue. Ici on ne paye rien pour le loyer, pour l'électricité, la nourriture n'est pas très chère."

Les constructions utilisent les matériaux disponibles, des bambous pour la structure, du carton pour isoler du froid, le plastique des serres pour imperméabiliser, le tout fixé au sol par le sable de la forêt. Autour des tentes, les objets indispensables: "les chaises, les casseroles, les bidons propres $^{24 "}$. Même sans travailler, on peut y survivre avec un minimum de ressources.

La chabola fonctionne par foyers. Dans le groupe de Daouda, environ 8 personnes se retrouvent tous les soirs autour du riz au poisson: "Tous les soirs on mange ensemble, et on parle des problèmes qu'il y a eus dans la journée, c'est comme la famille quoi." Ceux qui partagent le même repas cotisent entre 3 et 5 euros par semaine et se répartissent les corvées d'eau, de vaisselle, de cuisine et de bois. Parfois, un membre du groupe revient avec un gros sac de légumes qu'il a volés dans les champs. Tous les jours, l'un d'eux va pêcher dans l'embouchure du río Tinto. Ceux qui travaillent payent à manger à ceux qui n'ont pas trouvé de patron. Beaucoup d'hommes 
ont des partenaires roumaines ou marocaines qui viennent leur rendre visite le soir et même dormir sur place, les fermes étant généralement interdites aux hommes qui n'y travaillent pas. À la tombée du soir, lorsque de petits groupes

Ainsi, les faibles liens

établis pendant la saison

ne se transforment pas

en une amitié plus durable

qui pourrait pousser

un groupe de travailleurs

à faire la roue saisonnière

ensemble ou à s'allier pour

faire valoir leurs droits. jouent aux dames sur des cartons en écoutant sur leur portable la musique du pays, il apparaît évident que la chabola est un espace social à part entière, un refuge qui fournit dans cette zone le seul logement possible pour quelqu'un de passage et qui n'a pas de travail ${ }^{25}$. Elle est aussi un lieu où s'échangent des informations sur les campagnes, les papiers, le travail.

En juin 2010, alors que je rentrais en France en voiture, j’ai emmené Daouda et trois de ses amis à Lérida. Le jour du départ, alors qu'il n'y avait pourtant jamais mis les pieds, Daouda connaissait déjà les différentes possibilités de logement qui s'offraient à lui : "Y a une chabola mais les policiers ils emmerdent trop les gens. Y a le centre de Lérida, mais il faut payer une maison, donc avoir du travail. Je vais me cacher dans ce village et y aura pas de problèmes. Si je croise celui qui m’a parlé de ce village, il va me dire où il vit, comment vivent les autres, je vais faire comme eux. Ça s'arrête là. Mais dire que je vais descendre chez lui, non." Ainsi, les faibles liens établis pendant la saison ne se transforment pas en une amitié plus durable qui pourrait pousser un groupe de travailleurs à faire la roue saisonnière ensemble ou à s'allier pour faire valoir leurs droits. "On se retrouve là, c'est Dieu pour tous, chacun pour soi. À chaque campagne on a de nouvelles têtes, lui c'est mon ami de Huelva", dit Daouda. Lobligation de se déplacer, la précarité de leur installation toujours menacée par une éventuelle destruction expliquent qu'"on ne peut rien organiser du tout, tu vois, tout le monde s'en va tout le temps. On ne peut rien organiser d'important". Un jour, en mai 2010, alors qu'après l'annonce d'une prochaine destruction, un tracteur est entré dans le périmètre de la chabola, Abdou a explosé : "Tu vois, comment tu veux apprendre? On n'a pas le temps d'apprendre l'espagnol, on a trop de choses à penser, les problèmes du travail, les problèmes des papiers, les problèmes du pays, la tête est chaude, $y$ a rien qui rentre. Tu n'as pas le temps parce que l'esprit n'est pas tranquille."

Malgré tout, il existe des ancrages dans le transit. Dans la chabola de la madre, on peut être "ancien" ou "nouveau", et après six mois d'absence à ramasser les fruits ou les olives plus au Nord, on a tendance à se réinstaller dans le même coin de forêt. Même si les travailleurs sont très mobiles, leur rotation est elle aussi canalisée. En 2010, ne trouvant pas de travail, Daouda, tenté de se rendre à $40 \mathrm{~km}$ de là, finit par abandonner son projet: "Je ne veux pas aller jusqu'à Cartaya, être encore nouveau dans un nouvel endroit."

Dans un sens, ces trois ans de transit, pendant lesquels il semble au premier abord qu'ils ne peuvent finalement quattendre et se préparer au mieux au dépôt du dossier d'arraigo, ne sont pas des années où le temps reste suspendu. De 2009 à 2011, la roue saisonnière de Daouda s'est stabilisée. Il connaît des patrons à Jaén, Huelva et Lérida, et il essaie de concilier au mieux ses obligations envers ces derniers malgré le chevauchement des saisons. Il sait comment trouver du travail, contrairement aux premières années où, par manque de réseau, de savoir-faire et de maitrise de l'espagnol, il pouvait rester huit ou neuf mois sans travailler.

\section{Comment la recherche de papiers bride le salariat}

Pour les travailleuses sous contrat comme pour les sans-papiers, chercher à obtenir un statut juridique plus stable, c'est entrer dans la définition que l'État espagnol tente de donner du travailleur étranger et donc accepter une canalisation juridique, géographique et sectorielle. L'illégalisation, qui oblige pendant trois ans à faire la roue saisonnière, remplit finalement plus ou moins la même 
fonction que la canalisation mise en place ultérieurement via la contratación en origen. Sans papiers ou sous contrat, la mobilité géographique et sectorielle de ces ouvriers agricoles est tout autant canalisée. Pour les étrangers aux statuts juridiques les plus précaires, ces espaces-temps que sont les saisons fonctionnent en réalité comme des nasses reliées entre elles par un réseau de bus qui permet de se rendre d'une campagne à une autre, mais rarement de sortir du circuit de la roue saisonnière par peur du contrôle. Au point de pousser certains à ne plus bouger. Mohamed considère que c'est quand il a "commencé à chercher les papiers que le chemin a commencé à se serrer. C'est comme si tu faisais une longue route pour arriver à une porte fermée, et derrière la porte y a des merveilles, mais tu peux pas les sentir. Si la route est longue, tu n'as pas la force et tu n'as pas envie de faire demi-tour. Avant, quand je ne cherchais pas les papiers, je m'en foutais, je bougeais dans le Nord pour travailler, et puis je revenais ici à Huelva pour les papiers. Mais ici ils m'ont dit: 'Tu es fou, la gare à Huelva est pleine de poulets, il y a beaucoup de contrôle', et donc j'ai arrêté de bouger, je suis resté ici pour faire les papiers. Et c'est ça qui fait que ça n'avance pas. [...] C'est un peu dur de vivre au point mort pendant longtemps. [...] Toi tu es libre, moi mes ailes, elles sont cassées ${ }^{26}$."

L'idée de voir filer le temps sans que rien n'avance est reprise par tous les interlocuteurs: "Je perds mon temps, je vieillis, ça c'est pas bon”, "On peut pas rester assis ici", "J'attends, je veux plus y penser, sinon tu attends tout le temps et ça te rend fou"...

Finalement, il semble que ce ne soit pas tant ce à quoi les papiers donnent accès qui confère une telle valeur à la régularisation, mais les souffrances, la résistance et l'attente endurées pour les obtenir. L’avancée de la situation juridique devient une idée fixe. À tel point qu'à l'hiver 2010, lorsqu'un jeune Malien est mort asphyxié dans sa cabane par la fumée d'un brasero, Daouda a commenté l'événement ainsi : "C'est triste, il avait la cita ${ }^{27}$ à ce qu'on m'a dit." Le racisme auquel ils sont confrontés implique un rejet de la langue.

Le rapport au temps, à l'avenir est incertain. Le futur est compliqué. Selon Mohamed, dans un tel contexte, ce serait de ne pas devenir fou qui serait anormal. Pour lui, Huelva s'apparente à un piège : "C'est comme si tu avais marché longtemps dans le désert et tu as trouvé un lac salé... Et tu obliges ton cerveau à filtrer cette eau-là. Et ça te grille la tête. Et tu veux pas retourner chercher de l'eau parce que c'est de la souffrance aussi. Alors tu restes. Hassan aliya, c'est mieux pour moi."

Devant cette expérience partagée, se forme une sorte de solidarité, d'intégration de la galère, qui va au-delà des appartenances communautaires. À Mohamed qui dit qu'il n'en peut plus, on répond: "La porte est ouverte, ça va aller, petit à petit avec la patience." La crise économique met des entraves au processus de stabilisation des étrangers arrivés en Espagne après 2006. Elle fragilise également ceux qui sont arrivés plus tôt. Parmi ceux qui habitent la forêt, beaucoup ont la carte de résidence de cinq ans. C'est ce qui fait dire à certains que dans ce contexte de crise qui empêche la mobi-

A contrario,

les statuts précaires permettent de contrôler la mobilité des travailleurs et répondent aux impératifs contradictoires qu'une saison longue impose aux employeurs : garder la main-d'œuvre sur place. lité vers un autre secteur, il n'y a plus vraiment de différence entre celui qui a des papiers et celui qui n'en a pas. On dit : "J'ai des papiers espagnols mais je veux les changer, ils ne valent rien. Ce sont des papiers de femmes comme on dit ici", ou encore : "Les papiers ça reste de l'eau, parce qu'il n'y a pas de travail."

Finalement à Huelva, comme dans les Bouches-duRhône, l'obtention d'un statut juridique plus stable complique l'accès au marché du travail agricole. A contrario, les statuts précaires permettent de contrôler la mobilité des travailleurs et répondent aux impératifs contradictoires qu'une saison longue impose aux employeurs : garder la main-d'œuvre sur place pendant la saison sans risque de la voir déserter tout en garantissant son départ lorsque la campagne s'achève. 